\title{
Computing Convex Layers of a Dynamic Point Set
}

\author{
Sanjib Sadhu and Niraj Kumar
}

\begin{abstract}
The convex layers of a given point set can be computed by iterative process of finding convex hull after discarding the points of already computed convex hull. Computation of convex layers has been widely studied in the static environment where the point set are fixed. In this paper, we propose an idea to compute set of convex layers in dynamic context. There exists an optimal time algorithm to solve the static version of the problem in $0(n \log n)$ time. However, to solve dynamic version of the problem the suggested algorithm requires $\mathrm{O}\left(n^{2}\right)$ time for a set of $n$ points.
\end{abstract}

Index Terms - Computational geometry, convex hull, convex layers, incremental algorithm, tangent.

\section{INTRODUCTION}

The set of convex layers (or simply convex layers) can be computed by an iterative process of computing convex hull, after discarding points of already computed convex hull. It is one of the widely studied computational geometry problem, because of its wide area of applications. The convex layers can be applied to design a robust estimator [1]. Tukey [2] suggested a procedure called "peeling", for eliminating outliers. Another application of convex layers has been suggested by Lee et al. in the half plane range query problem [3]. Recently, Liew [4] suggested application of convex layers to TSP problem. Much effort has been applied to compute the convex layers of fixed point set. There exist an algorithm [5] to compute the convex layers for fixed point set in optimal time, i.e., in $O(n \log n)$ time. However, there exist instances, where point set is not fixed and points can be inserted or deleted at any time. In this paper, we suggest an algorithm to compute the convex layers in dynamic context. The convex layers can be computed in $O\left(n^{2}\right)$ time by applying the proposed approach.

To compute a convex layers from static point set, a brute force approach can be applied, which involves applying an optimal convex hull algorithm, say [6], repeatedly for each layer, thus it requires $O\left(n^{2} \log n\right)$ time. Silverman et al. reduced time complexity to $O\left(n^{2}\right)$ [7]. Chazelle [5] proposed algorithm to compute the convex layers in optimal time. In [5] the deletion process batched to give $O(n \log n)$ time algorithm.

This paper is organized as follows. Some preliminaries are presented in Section II. Detailed description of proposed algorithm with its analysis is presented in Section III. Finally,

Manuscript received July 13, 2014; revised October 10, 2014.

Sanjib Sadhu is with the Department of Computer Science, National Institute of Technology, Durgapur, India 713209 (e-mail: sanjibsadhu411@gmail.com).

Niraj Kumar was with the National Institute of Technology, Durgapur, India 713209. He is now with the Department of Computer Science, Dronacharya College of engineering, Gurgaon, Haryana (e-mail: nirajcse08@gmail.com). in Section IV, we conclude with possible future works.

\section{PRELIMINARIES}

A subset $S$ of the plane is called convex [8] if and only if for any pair of points $(p, q) \in S$ the line segment $\ell s(p, q)$ is completely contained in $S$. The Convex Hull $C H(S)$ of a point set $S$ is the smallest convex set that contains $S$ and is represented by set of vertices that defines hull edges.

For a given 2-dimensional planar point set $S=$ $\left\{s_{1}, s_{2}, \ldots, s_{n}\right\}$, its Convex Layers, denoted by $C L$, can be visualized as set of convex hulls computed by iterative procedure of computing convex hull and discarding the points on already computed convex hull (Refer to Fig. 1). In this paper, to represent a convex hull $C L_{i} \in C L$, we use counterclockwise $(\mathrm{CCW})$ sequence of vertices on convex hull $C L_{i}$. For a point $p \in C L_{i}$ (Refer to Fig. 1), point p.prev and $p$.next represents point previous to $p$ and the point next from $p$, respectively (in CCW order).

\section{Algorithm}

The point set is dynamic, so, at any time some point may be inserted or deleted from point set. Computing convex layers in dynamic context requires insertion as well as deletion of points to $C L$. In this section we give the detailed description of two procedures viz.: Insert() and Delete() to accomplish insertion and deletion of points, respectively.

Assumption: All the points are in general position, i.e. no three points are collinear.

Assumption: The layers $C L_{i} \in C L$, are numbered outward to inward. Outermost layer is $C L_{1}$ and innermost is $C L_{k}$, where $k$ be the number of layers in $C L$.

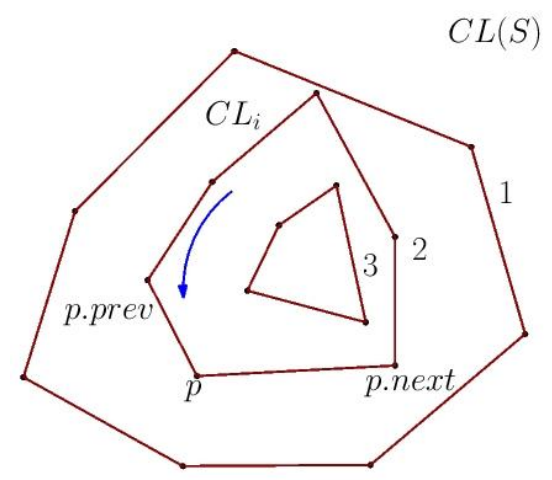

Fig. 1. Convex layer set $C L$.

Definition: The region (here unbounded) is defined as the minor sector formed by two rays. In the Fig. 2, region $R\left(p_{1}, p_{2}, p_{3}\right)$ is the minor sector formed by rays $r_{1}$ (passing through point $p_{1}$ ) and $r_{2}$ (passing through point $p_{3}$ ) such that both rays have common fixed point $p_{2}$. 


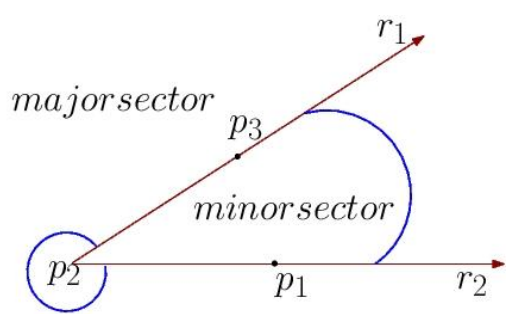

Fig. 2. Region.

The line passing through point $p$ and $q$ is represented by $\ell(p, q)$. However, the line segment with the end points $p$ and $q$ is represented by $\ell s(p, q)$.

Points $p$ and $q$ are said to be visible if $\ell s(p, q)$ does not intersects any other line segment or does not passes through any other point. Hence, in Fig. $3, p_{1}$ and $q$ are visible, whereas $p$ and $q$ are not visible.

We say, a point $q$ is lying outside layer $C L_{i}$, if it is lying outside $C L_{i}$ but not outside $C L_{i-1}$.

\section{A. Algorithm to Insert a Point $q$ to $C L$}

Algorithm 3 inserts an incoming point to $C L$. To insert an incoming point $q$ to convex layer set $C L$, first it is required to determine the layer $C L_{i}$ to which $q$ will be inserted, the Algorithm 1 serves this purpose. Insertion of a point to $C L_{i}$ requires removal of chain lying under the wedge formed by two tangents from the point $q$, i.e., the part of $C L_{i}$ visible from $q$. Consider Fig. 3, insertion of point $q$ to $C L_{i}$ requires removal of the visible part of $C L_{i}$, i.e., m.prev to $p_{1}$. A point is said to be lower (resp. upper) tangent point, denoted by $v_{l}$ (resp $v_{u}$ ), if it is first (resp. last) point of the chain in $\mathrm{CCW}$ order. Algorithm 2 computes the two tangent points.

\section{1) Algorithm 1: Find layer}

To determine the layer $C L_{i} \in C L$, to which point $q$ is to be inserted, a search is performed outward (from $C L_{1}$ ) to inward (to $C L_{k}$ ). While inspecting a layer $C L_{i}$, a point $p \in C L_{i}$ is chosen randomly. If point $q$ is not lying in region, $R$ (p.prev, p,p.next), then the point is certainly outside the layer $C L_{i}$. For instance, in Fig. 3 for point $p_{1}$ the point $q$ lies outside the region $R(p . p r e v, p, p . n e x t)$. Hence, point $q$ lies outside layer $C L_{i}$ (It is important to note that layer $C L_{1}$ to $C L_{i-1}$ has already been examined and search has failed.) Otherwise, point may be lying inside or outside the layer. A binary search is performed to contract the region and every time either p.prev or p.next is updated until both become adjacent depending on whether $q$ is on the left or right of $\ell\left(p, p_{m}\right)$, where $p_{m}$ is midpoint of chain $p$. next to p.prev. If the line segment $\ell s(q, p)$ intersects $\ell s(p$.next,p.prev) then point $q$ is outside the layer $C L_{i}$ (layer being examined), otherwise next layer checked to find out if $q$ is lying outside.

\section{Algorithm 1: Find Layer $(q, C L)$}

Input: Point $\mathrm{q}$ and convex layers $C L$, say $k$ is the number of layers in $C L$.

Output: The convex layer $C L_{i}$ such that $q$ lies outside $C L_{i}$.

1) $i \leftarrow 1$

2) while $(i \neq k+1)$ do

3) if ( $i=k$ and $C L_{k}$ contains less than three points)

4) Return $k$

5) else

6) select a point $p \in C L_{i}$

7 ) if ( $q$ is not in region $R(p . p r e v, p, p . n e x t)$ )
8) Return $i$

9) else

10) repeat

11) $m:=(p . n e x t+p . p r e v) / 2$

12) if ( $q$ is to left of $\ell\left(p, p_{m}\right)$ )

13) p. next: $=m$

14) else

15) $p$. prev: $=m$

16) until (p.next \& p.prev are not adjacent)

$17)$ if $(\ell s(q, p)$ intersects $\ell s(p . n e x t, p . p r e v))$

18) return $i$

19) else

20) $i++$

21) Return $k+1 / / q$ is inside $C L_{k}$

Let $n$ be the total number of points in the point set $S$ and $n_{i}$ be the number of points in the layer $C L_{i}$. Let $k$ be the number of layers in convex layer set $C L$. Algorithm 1 determines the layer to which incoming point should be inserted. The layers are considered in outward to inward fashion. Inspection of each layer, $C L_{i}$, takes $\log \left(n_{i}\right)$ time. So, in the worst case all layers inspected, hence, time to compute layer is

$$
\begin{gathered}
\log \left(n_{1}\right)+\log \left(n_{2}\right)+\ldots+\log \left(n_{k}\right) \\
\leq n_{1}+n_{2}+\ldots+n_{k}=n
\end{gathered}
$$

Hence, it is clear that correct layer to which incoming point should be inserted can be found in $O(n)$ time.

\section{2) Algorithm 2: Compute tangent}

A line segment from point $q$ to a point $v \in C L_{i}$ is said to be a tangent from $q$ to $C L_{i}$, if both the points $v$.prev and $v$. next lie on same side of $\ell(q, v)$. If $v$.prev and $v$. next lies on the right (resp. left) of $\ell(q, v)$ then $v$ is said to be lower (resp. upper) tangent point, denoted by $v_{l}$ (resp. $v_{u}$ ).

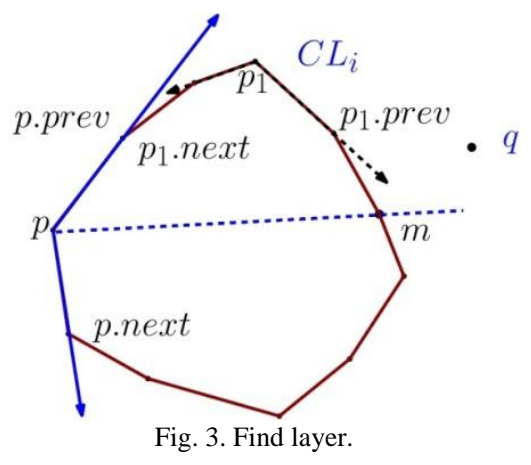

Let $q$ be a point outside layer $C L_{i}$. Then, two points $v$ and $v_{1}\left(\epsilon C L_{i}\right)$ are said to be of similar nature if i). $\ell_{s}(q, v)$ intersects $\ell s(v . p r e v, v . n e x t)$ and $\ell_{s}\left(q, v_{1}\right)$ intersects $\ell s\left(v_{1}\right.$.prev, $v_{1}$. next), or ii). Neither $\ell s(q, v)$ intersects $\ell s(v . p r e v, v . n e x t) \quad$ nor $\quad \ell s\left(q, v_{1}\right) \quad$ intersects $\ell s\left(v_{1}\right.$. prev, $v_{1}$.next $)$

A point, say $v_{2}\left(\epsilon C L_{i}\right)$, is chosen randomly and if it is not a tangent point then select another point, say $v_{3}$, at $n / 2$ distance from $v_{2}$. If both the points are of same nature, then chain $v_{2}$ to $v_{3}$ does not contain any tangent point, whereas chain $v_{3}$ to $v_{2}$ contains both the tangent points. Otherwise, both the chains $v_{2}$ to $v_{3}$ and $v_{3}$ to $v_{2}$ contains one tangent point (Refer Fig. 4).

In Algorithm 2, whether the randomly selected point is 
tangent or not can be checked in constant time. In the contraction step 9-13, binary search like procedure followed, every time length of chain reduced to $n / 2$. As the number of points $\left(n_{i}\right)$ in layer $C L_{i}$ is $O(n)$. Thus, a tangent point can be computed in $O(\log n)$ time. Hence, it is clear that two tangent points can be computed in $O(\log n)$ time.

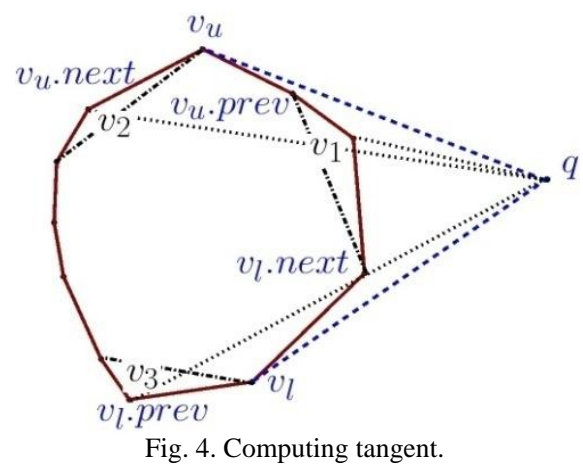

\section{3) Algorithm 3: Insert point}

To insert a point, say $q$, to convex layer $C L_{i}$, the chain $v_{l}$ to $v_{u}$ must be added to next inner layer, which in turn may require inner layer(s) to be reconfigured (Refer to Fig. 5).

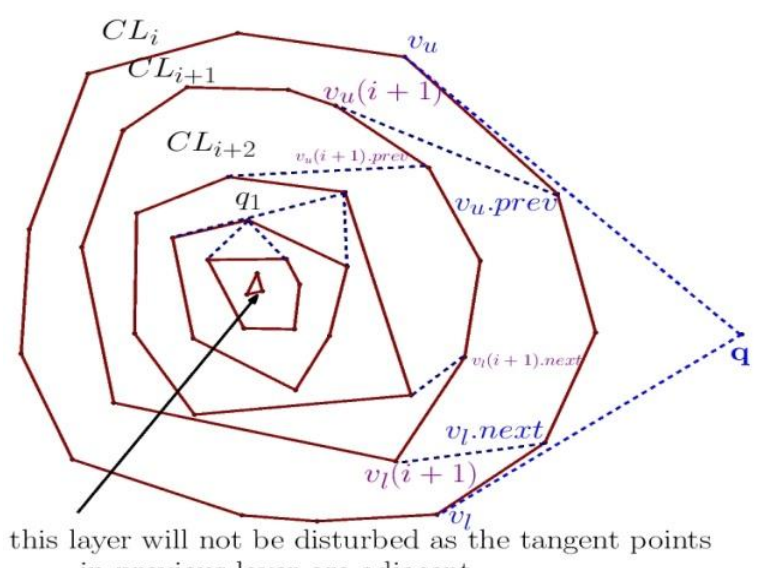
in previous layer are adjacent

Fig. 5. Inserting a point to $C L$.

The Algorithm 2 can be easily modified to compute either upper tangent only (i.e. procedure Compute Upper Tangent() in Algortihm 3) or to compute lower tangent only (i.e. procedure Compute Lower Tangent() in Algortihm 3). A convex hull edge is represented by $e(u, v)$, where $u$ and $v$ are two consecutive vertices on the convex hull.

\section{Algorithm 2: Compute Tangent $\left(q, C L_{i}\right)$}

Input: point $q$ and convex layer $C L_{i}$ $C L_{i}$

Output: upper $\left(v_{u}\right)$ and lower tangent point $\left(v_{l}\right)$ from $q$ to

1) $n:=$ no. of points in $C L_{i}, S_{i}:=$ points of $C L_{i}$

2) while (two tangent points not computed) do

3) select a point $v \in S_{i}$

4) if( $v$. prev and $v$.next lies on the right of $\ell(q, v))$

5) return $v$ as lower tangent point $\left(v_{l}\right)$

6) else if $(v$.prev and $v$. next lies on the left of $\ell(q, v))$

7) return $v$ as upper tangent point $\left(v_{u}\right)$

8) else

9) select a point $v_{1}$ at $n / 2$ distance from $v$

10 ) if points $v$ and $v_{1}$ are of similar nature

11) discard chain $v$ to $v_{1}$
12) $S_{i}:=$ points of chain $v_{1}$ to $v$

13) go to step 3

14) else

15) $S_{1}:=$ points of chain $v$ to $v_{1}$

16) $S_{2}:=$ points of chain $v_{1}$ to $v$

17) go to step 3 with $S_{1}$

18) go to step 3 with $S_{2}$

\section{Algorithm 3: Insert Point $(q, C L)$}

Insert a point in the convex layer set while maintaining the convexity property. It assumes that at least three points are already arrived.

Input: $q$ is the point to be inserted to the convex layers $C L$ with $k$ layers.

Ouptut: Convex layers set $C L$ after insertion of point $q$.

1) $i=$ FindLayer $(q, C L)$

2) if $i=k+1$ then do

3) $C L_{k+1}=q / /$ new layer added

4) return $C L$ with $k+1$ layers

5) if $i=k$ and $C L_{k}$ contains less than three points

6) $C L_{k}=C L_{k} \cup q$

7) return $C L$

8) ComputeTangent $\left(q, C L_{i}\right)$

9) if $v_{l} \& v_{u}$ are adjacent

10) connect $q$ with $v_{u}$ and $v_{l}$

11) remove edge $e\left(v_{l}, v_{u}\right)$

12) return $/ / C L_{i+1}$ not disturbed

13) remove chain $\mathrm{v}_{\mathrm{l}}$. next to $\mathrm{v}_{\mathrm{u}}$. prev from $C L_{i}$

14) remove edges $\mathrm{e}\left(v_{l}, v_{l}\right.$. next) and e $\left(v_{u}\right.$. prev, $\left.v_{u}\right)$

15) connect $q$ with $v_{u}$ and $v_{l}$ to complete $C L_{i}$

16) $i=i+1$

17) while $(i \neq k)$ do

18) $v_{u}(i+1)=$ Compute Upper Tangent $\left(C L_{i+1}\right.$, $v_{u} \cdot$ prev)

19) $v_{l}(i+1)=$ Compute Lower Tangent $\left(C L_{i+1}\right.$, $v_{l}$. next)

20) if $v_{l}(i+1) \& v_{u}(i+1)$ are adjacent

21) connect $v_{u}$. prev with $v_{u}(i+1)$ and $v_{l}$. next

22) with $v_{l}(i+1)$

23) remove edge $e\left(v_{l}(i+1), v_{u}(i+1)\right)$

24) return $/ /$ next inner layer not disturbed

25) remove chain $v_{l}(i+1)$. next to $v_{u}(i+1)$. prev from $C L_{i+1}$

26) remove edges $e\left(v_{l}(i+1), v_{l}(i+1)\right.$. next)

27) \& $e\left(v_{u}(i+1) \cdot \operatorname{prev}, v_{u}(i+1)\right)$

28) connect $v_{u}$.prev with $v_{u}(i+1) \& v_{l}$. next with

29) $v_{l}(i+1)$

30) $v_{u}$.prev $:=v_{u}(i+1) \cdot$ prev

31) $v_{l}$. next $:=v_{l}(i+1)$. next

32) $i:=i+1$

33) $C L_{i}$ is chain $v_{u}$. prev to $v_{l}$. next

To insert an incoming point $q$ the layer, say $C L_{i}$, to which it should be inserted can be computed in $O(n)$ time and two tangent points to the layer $C L_{i}$ can be found in $O\left(\log n_{i}\right)$ time. With this information, point $q$ can be inserted to layer $C L_{i}$ in constant time. Consequently, inner layer(s) may require to be modified. In the worst case, the point inserted to outer layer and all inner layers required to be modified, in that case running time is: 
$\left.O(n)+O\left(\log n_{1}\right)\right\}$ updating outermost layer $\left.+O\left(\log n_{2}\right)+\cdots+O\left(\log n_{k}\right)\right\}$ updating all inner layers

$$
\begin{gathered}
\leq O(n)+O\left(\log n_{1}\right)+\left(n_{2}+n_{3}+\cdots+n_{k}\right) \\
\leq O(n)
\end{gathered}
$$

Hence, a single point can be inserted in $O(n)$ time.

\section{B. Algortithm to Delete a Point from $C L$}

Deletion of a point $q$ from convex layer $C L_{i}$ may result into reconfiguration of inner layer(s), whereas outer layers remain unaltered. Algorithm 4 deletes a point $q$ from $C L$ and reconfigures $C L$, so that convexity is maintained. Deletion involves finding tangents and updating inner layer(s) if required (Refer Fig. 6).

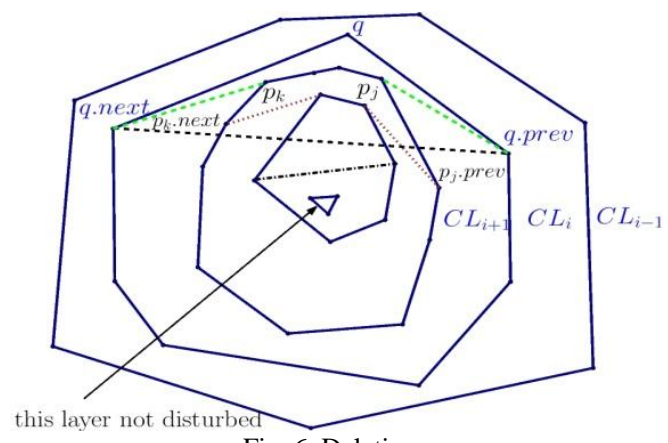

Fig. 6. Deletion.

Deletion of a point (or chain) from layer $C L_{i}$, may require inclusion of a part of layer $C L_{i+1}$ to layer $C L_{i}$ (Refer Fig. 6).

Candidate list CList stores points of $C L_{i+1}$ that are to be added to $C L_{i}$.

In step 7 of Algorithm 4, to maintain $\mathrm{CCW}$ order, points added to CList after q. prev and before q. next.

A point (or chain) can be added to or removed from layer $C L_{i}$ in constant time. The expensive step is computation of tangents. By applying Algorithm 2, a tangent can be computed in $O(\log n)$ time. In the worst case, after deletion of a point from outermost layer, all the inner layers are required to be modified. Thus, tangent computed for all inner layers,

$$
\begin{gathered}
\log \left(n_{2}\right)+\ldots+\log \left(n_{k}\right) \\
\leq n_{1}+n_{2}+\ldots+n_{k}=n
\end{gathered}
$$

Thus, the time required to delete a point is $O(n)$.

\section{Algorithm 4: Delete $\left(q, C L_{i}\right)$}

Input: $q$ is the point to be deleted from $C L_{i}$ and $k$ is number of layers in $C L$.

Output: Convex layers set $C L$ after deletion of point $q$.

1) Candidate list CList initialized as an empty list.

2) $i:=1$

3) repeat

4) Compute tangents from q.prev and q. next to $C L_{i+1}$ lying to the right of the line $l$ (q.prev, q. next), say $p_{a}$ and $p_{b}$ be tangent points, respectively. If no such points found, return.

5) The points lying on the chain $p_{a}$ to $p_{b}$ are added to CList in order.

6) if CList is empty //CL $L_{i+1}$ not disturbed

Remove $q$ from $C L_{i+1}$

Return
7) else

$$
\begin{aligned}
& C L_{i+1}:=C L_{i+1}-C \text { List } \\
& C L_{i}:=C L_{i}-\{q\} \\
& C L_{i}:=C L_{i} \cup \text { CList } \\
& \text { q.next }:=\text { LList }[\text { last].next } \text { q.prev }:=\text { CList }[\text { first].prev } \\
& \text { empty CList } \\
& i=i+1
\end{aligned}
$$

8) while $(i \neq k)$

Hence, it is clear that a single point can be inserted or deleted in $O(n)$ time. Algorithm 3 and Algorithm 4 can be used to compute the convex layers in the dynamic context in $O\left(n^{2}\right)$. However, the amortized time complexity will be much lower.

\section{CONCLUSION}

In this paper we have presented an idea to compute convex layers in dynamic environment. The primary requirement is to allow insertion and deletion at any instant. Time complexity of proposed algorithm is $O\left(n^{2}\right)$. To reduce the running time for insertion and deletion will be our future work.

\section{REFERENCES}

[1] M. I. Shamos, "Computational geometry," Ph.D. thesis. Dept. of Computer Science, Yale University, 1978.

[2] P. J. Huber., "Robust statistics: a review," Annals of Mathematical Statistics, vol. 43, no. 3, pp. 1041-1067, 1972.

[3] B. Chazelle, L. J. Guibas, and D. T. Lee, "The power of geometric duality," in Proc. 24th IEEE Annual Symposium Foundations of Computer Science, 1983, pp. 217-225.

[4] S. Liew, "Introducing convex layers to the Traveling Salesman Problem," The Computing Research Repository, 2012.

[5] B. Chazelle, "On the convex layers of a planar set," IEEE Transactions on Information Theory, vol. IT-31, pp. 509-517, 1985.

[6] R. L. Graham, "An efficient algorithm for determining the convex hull of a finite planar set," Information Processing Letter, vol. 1, pp. 132-133, 1972.

[7] P. J. Green and B. W. Silverman, "Constructing the convex hull of a set of points in the plane," Computer Journal, vol. 22, pp. 262-266, 1979.

[8] M. D. Berg, O. Cheong, M. V. Kreveld, and M. Overmars, Computational Geometry: Algorithms and Applications, 3rd ed., Springer-Verlag TELOS Santa Clara, CA, USA, 2008.

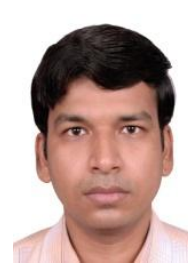

Sanjib Sadhu has done his B.E. degree in computer science and engineering from NIT Durgapur and M.Tech. degree in computer science and engineering from N.I.T. Durgapur. Currently he is working with the Department of Computer Science and Engineering at NIT Durgapur as an assistant professor. His research areas include algorithm design and computational geometry.

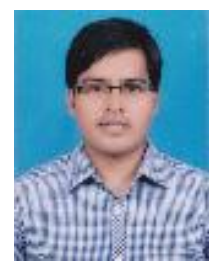

Niraj Kumar got the B.E. degree in computer science and engineering from MDU Rohtak and M.Tech degree in computer science and engineering from N.I.T. Durgapur. Currently he is working with the Department of Computer Science and Engineering at Dronacharya College of Engineering Gurgaon as an assistant professor. His research areas include algorithm design and computational geometry. 